\title{
LEVANTAMENTO DA FAUNA DE ABELHAS SILVESTRES (HYMENOPTERA, APOIDEA) NA REGIÃO DA "BAIXADA MARANHENSE": VITÓRIA DO MEARIM, MA, BRASIL ${ }^{1}$
}

\author{
Patricia M.C. de ALBUQUERQUE ${ }^{2}$, Rosilene da G. FERREIRA ${ }^{3}$, Márcia M.C. \\ RÊGO², Cláudia S. dos SANTOS ${ }^{4}$, Ciclene M. S. de BRITO $^{5}$
}

RESUMO - Estudou-se durante um ano a fauna de abelhas de uma região da Baixada maranhense em Vitória do Mearim - MA. Foram feitas coletas mensais com auxilio de redes entomológicas e armadilhas de cheiro, no periodo de um ano, totalizando 288 horas de amostragem. Um total de 839 indivíduos de 38 espécies de abelhas pertencentes às familias Apidae, Megachilidae, Halictidae, Andrenidae e Colletidae foram coletadas nas flores e 72 individuos (11 espécies) de Euglossinae em armadilhas com íscas-odoríferas. Scaptotrigona flavisetis Moure, Trigona pallens Cockerell e Apis mellifera Linnaeus foram as espécies mais abundantes na área. A sazonalidade foi variável de acordo com as diferentes espécies de abelhas. S. flavisetis foi observada em maior número em janeiro e outubro, $T$. pallens em janeiro e fevereiro e $A$. mellifera em abril. Das abelhas coletadas em armadilhas, Euglossa (E.) cordata e $E$. (E.) gr. modestior foram as mais abundantes e Eucaliptol foi a ísca odorifera que recebeu maior número de visitas.

Palavras-chave: Apoidea, sazonalidade, visitantes florais, íscas odoriferas

Survey of the Wild Bees (Hymenoptera, Apoidea) of the "Maranhão Lowland": Vitoria do Mearim, MA, Brazil

ABSTRACT - The bee fauna of a restrict area of Lowland Region of Maranhão was studied. Monthly samples were performed with entomological net and baits trap with attractive compounds, totalling 288 hours of sampling. A total of 839 individuals belonging to 38 species of the bee families Apidae, Megachilidae, Halictidae, Andrenidae and Colletidae were collected on flowers and 72 individuals (11 species) of Euglossinae in baits trap. Scaptotrigona flavisetis Moure, Trigona pallens Cockerell and Apis mellifera Linnaeus were most frequent species in the area. Bee frequencies showed various patterns of seasonality, i.e. the period in which most bees of $S$, flavisetis were observed were January and October, T. pallens in January and February and A. mellifera in April. Euglossa (E.) cordata and $E$. (E.) gr. modestior were the most frequent species collected in baits traps and Eucaliptol was the most attractive compound.

Key-words: Apoidea, seasonality, flower-visiting, chemical baits

\section{INTRODUÇÃo}

Os levantamentos de abelhas e flora associada, utilizando-se amostragens padronizadas, iniciaram- se no Brasil com o trabalho de Sakagami et al. (1967), e a partir daí vários inventários em áreas naturais $\mathrm{e}$ alteradas de diferentes ecossistemas têm sido realizados: Sakagami \&

\footnotetext{
'Trabalho executado com auxílio financeiro do Conselho Nacional de Desenvolvimento Científico e Tecnológico (CNPq), proj. N ${ }^{\circ}$ 400675/91-9; Fundação de Amparo à Pesquisa do Estado do Maranhão (FAPEMA), proj. N $N^{\circ} 119 / 92$ e da Companhia Vale do Rio Doce (CVRD).

${ }^{2}$ Departamento de Biologia, Universidade Federal do Maranhão-UFMA, Av, dos Portugueses, Campus Universitário do Bacanga, São Luís, MA, CEP: 65080-040

${ }^{3}$ Bolsista de Iniciação científica $-\mathrm{CNPq}{ }^{\circ}$ 800717/91-0

${ }^{4}$ Bolsista do PET-CAPES-UFMA

${ }^{5}$ Bolsista do PIBIC-CNPq-UFMA
} 
Laroca (1971; 1974), Laroca et al. (1982), Cure (1983), Camargo \& Mazucato (1984), Bortoli \& Laroca (1990), Wittman \& Hoffman (1990), Taura \& Laroca (1991), Martins (1994, 1995), Silveira \& Campos (1995), Schlindwein (1998), Carvalho \& Bego (1995; 1996), Albuquerque \& Mendonça (1996), Gonçalves et al. (1996), Wilms et al. (1996), Albuquerque (1998), Rêgo (1998), Zanella et al. (1998).

O Estado do Maranhão, devido a sua posição geográfica peculiar (intermediário entre as regiões amazônicas, centro-oeste e nordeste) possui uma grande diversidade de formações vegetais, tais como manguezais, campos, dunas, restingas, cerrado, cerradão, caatinga/carrasco, babaçuais, floresta tropical úmida e floresta tropical seca (Brasil, 1991).

Este trabalho é parte do levantamento de abelhas que vem sendo feito no Maranhão, tendo sido precedido por estudos semelhantes realizados em mata secundária em São Luis (Rebêlo, 1995) e em Alcântara (Gonçalves et al., 1996.) e no cerrado de Barreirinhas (Albuquerque \& Mendonça, 1996; Rebêlo \& Cabral, 1997). Segundo Ab'Saber (1987), a Baixada Maranhense em seu sentido mais amplo, corresponde a importante faixa de planicies de nível de base, formadas a partir dos fundos de uma paleorreentrância regional da costa maranhense, parcialmente colmatada por depósitos fluviais, flúvio-lacustres e flúvio-marinhos. $\hat{E}$ objetivo nosso contribuir para o conhecimento da estrutura de comunidades de abelhas no Nordeste do Brasil. Neste trabalho estudamos a composição, a abundância relativa e a flutuação sazonal das diversas espécies de abelhas, fazendo-se uma comparação com outros levantamentos já realizados no Maranhão e em outras regiões do Brasil.

\section{MATERIAL E MÉTODOS}

Vitória do Mearim localiza-se na porção Ocidental do Maranhão com altitude de $18 \mathrm{~m}$, latitude $3^{\circ} 29^{\prime}$ e longitude 44³8' (Brasil, 1984), sendo a área de coleta paralela à ferrovia São Luís - Carajás, Km 142. Os niveis médios anuais de pluviosidade são em torno de $2000 \mathrm{~mm}$ concentrados entre dezembro e maio (Brasil, 1991). Do ponto de vista térmico caracteriza-se por apresentar máximas de $34^{\circ} \mathrm{C}$ e minimas de $27^{\circ} \mathrm{C}$ (Brasil, 1984).

É uma região da Baixada Maranhense, fortemente influenciada pela ação antrópica (queimadas e derrubadas) e sujeita a inundação periódica. A vegetação de Vitória do Mearim é principalmente secundária e arbustiva, com predominância da palmeira babaçu (Orbignia martiniana), sendo que a área especifica de coleta é uma capoeira, na qual a vegetação se modifica continuamente, tornando-se menor sua vegetação arbustiva.

As coletas foram feitas de dezembro de 1991 a novembro de 1992 a cada intervalo de 30 dias, das 13:00 
às 18:00 hs no primeiro dia e das 06:00 às 13:00 hs no dia seguinte, perfazendose um total de doze horas de coleta. Foram realizadas por duas pessoas, que percorriam o perímetro em sentido contrário; este percurso era realizado em uma hora passando-se cerca de dois minutos em cada planta. As abelhas foram coletadas com redes entomológicas padronizadas durante ou após as visitas às flores e colocadas em câmaras mortiferas à base de acetato de etila, acondicionadas em sacos plásticos e separadas por horário, planta coletada e data. Foram ainda usados iscasodoriferas de salicilato de metila, eugenol, vanilina e eucaliptol em armadilhas tipo "MELPAN Produtos Agricola Ltda" modificadas para coleta de machos de Euglossinae por Campos et al. (1989).

As espécies coletadas foram depositadas na coleção Entomológica do Departamento de Biologia da Universidade Federal do Maranhão.

A dominância de cada espécie foi calculada segundo o método de KATO et al. (1952), pelo qual são consideradas dominantes as espécies da amostra cuja abundância relativa apresenta o limite de confiança inferior maior que o limite de confiança superior para $K=0(0,36)$.

\section{RESULTADOS E DISCUSSĀO}

Foram coletadas sobre flores 839 abelhas de 36 espécies e 72 espécimens de Euglossinae (11 espécies) em iscas odoriferas (Tab. 1). Das 36 espécies encontradas, 20 foram consideradas predominantes $(52,6 \%$; Fig. 1): Apidae (11 espécies) com 685 individuos de um total de 696; Megachilidae (5 espécies) com 87 entre os 102 individuos; Halictidae (3 espécies) com 25 indivíduos entre 31 e Andrenidae (1 espécie) com 5 individuos. Na Figura 1 as espécies estão representadas em ordem descendentes de abundância (do topo para a base), de acordo com o número de indivíduos capturados. Scaptotrigona flavisetis Moure, Trigona pallens Cockerell e Apis mellifera Linnaeus, espécies mais abundantes, foram coletadas durante todo o ano, o que pode ser explicado devido ao caráter social dessas espécies, a ampla distribuição em diferentes habitats no Maranhão e presença de ninhos próximos à área de coleta.

Observou-se que não houve uma relação nítida entre os parâmetros climáticos e a atividade anual das abelhas, como também não foi observada uma variação nitida na abundância dos indivíduos e das espécies entre os períodos seco e chuvoso (Fig. 2). Apesar de que entre março e junho, meses em que ocorreram a maior riqueza de abelhas, coincide com o final do período chuvoso (diminuição da precipitação e umidade relativa; Fig. 2). Observamos um pico da atividade dos indivíduos em janeiro (133), devido principalmente à acentuada presença de Meliponini, especialmente, Scaptotrigona flavisetis (Fig. 2 e 3A). Durante a estação chuvosa, em dias năo chuvosos, é comum a coleta de 
Tabela 1. Espécies de abelhas e número de especimens coletados em Vitória do Mearim, MA, Brasil, Dez/1991 - Nov/1992.

\begin{tabular}{|c|c|c|}
\hline ESPÉCIE & Sobre Flores & Isca-odorifera \\
\hline ANDRENIDAE & 5 & \\
\hline Oxaea festiva Smith, 1854 & 5 & \\
\hline HALICTIDAE & 31 & \\
\hline Augochlorini & 2 & \\
\hline Augochlora (Augochlora) cf. caerulior Cockerell, 1900 & 2 & \\
\hline Augochlora sp. & 2 & \\
\hline Augochlora (Oxystoglossella) thalia Smith, 1879 & 9 & \\
\hline Augochloropsis sparsilis (Vachal, 1903) & 11 & \\
\hline Augochloropsis crassigena Moure, 1943 & 5 & \\
\hline APIDAE & 696 & 72 \\
\hline Eufriesea mussitans (Fabricius, 1787) & 3 & 3 \\
\hline Eulaema (Apeulaema) nigrita Lepeletier, 1841 & 7 & 19 \\
\hline Eulaema (Apeulaema) cingulata (Fabricius, 1804) & & 2 \\
\hline Eulaema (Eulaema) meriana (Olivier, 1789) & & 1 \\
\hline Euglossa (E.) cordata (Linnaeus, 1758) & 5 & 18 \\
\hline Euglossa (E.) townsendi Cockerell, 1904 & 1 & \\
\hline Euglossa (E.) gr. purpurea sp 1 & 1 & \\
\hline Euglossa (E.) gr. purpurea sp 2 & 5 & \\
\hline Euglossa (E.) gaianii Dressler, 1982 & & 7 \\
\hline Euglossa (E.) securigera Dressler, 1982 & & 2 \\
\hline Euglossa (E.) ignita Smith, 1874 & & 2 \\
\hline Euglossa (E.) mourei Dressler, 1982 & & 1 \\
\hline Euglossa (E.) modestior Dressler, 1982 & & 14 \\
\hline Exaerete smaragdina (Guérin-Meneville, 1845) & & 3 \\
\hline Melipona compressipes fasciculata Smith, 1854 & 4 & \\
\hline Tetragona dorsalis (Schwarz, 1938) & 5 & \\
\hline Trigona fuscipennis Friese, 1900 & 20 & \\
\hline Trigona pallens (Fabricius, 1798) & 152 & \\
\hline Trigona fulviventris Guérim, 1835 & 33 & \\
\hline Trigona spinipes (Fabricius, 1793) & 39 & \\
\hline Trigonisca sp. & 2 & \\
\hline Scaptotrigona flavisetis Moure, MS & 331 & \\
\hline Bombus brevivilus Franklin, 1913 & 14 & \\
\hline Apis mellifera Linnaeus, 1758 & 74 & \\
\hline MEGACHILIDAE & 102 & \\
\hline Hypanthidium sp. & 10 & \\
\hline Megachile sp. 1 & 15 & \\
\hline Megachile sp. 2 & 22 & \\
\hline Megachile sp. 3 & 2 & \\
\hline Megachile sp. 4 & 2 & \\
\hline Megachile (Pseudocentrum) sp. 1 & 1 & \\
\hline Megachile (Pseudacentrum) sp. 2 & 3 & \\
\hline Megachile (Pseudocentrum) sp. 3 & 1 & \\
\hline Megachile (Pseudocentrum) sp. 4 & 1 & \\
\hline Megachile (Sayapis) sp. 1 & 9 & \\
\hline Megachile (Sayapis) sp. 2 & 32 & \\
\hline Megachilini & 4 & \\
\hline COLLETIDAE & 4 & \\
\hline Hylaeus sp. & 4 & \\
\hline TOTAL GERAL & 838 & 72 \\
\hline
\end{tabular}




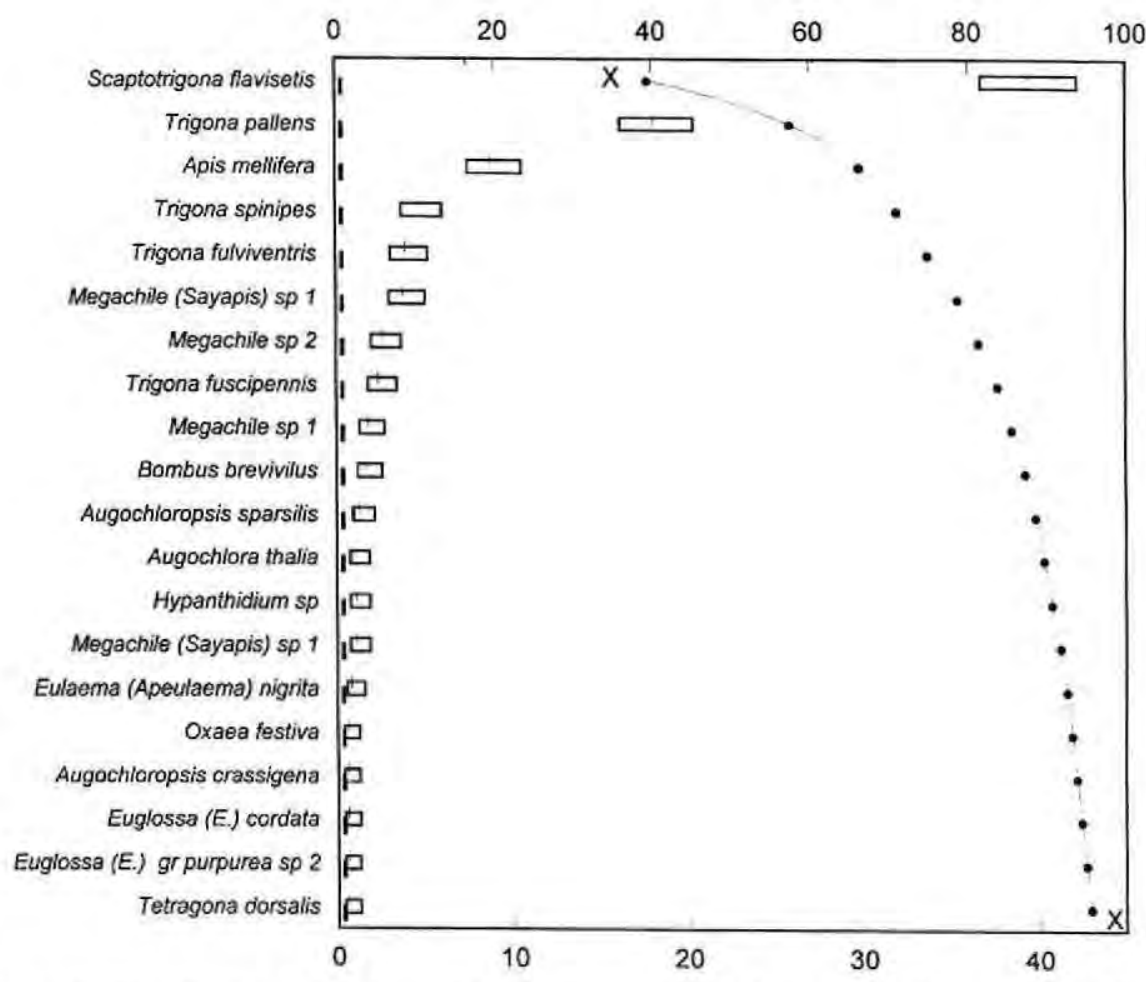

Figura 1. Abundância relativa das espécies predominantes de abelhas coletadas em visita às flores em Vitória do Mearim. Os limites de confiança sâo representados pelas extremidades dos retângulos horizontais e as barras verticais representam a razão de porcentagem (escala inferior); a linha tracejada vertical paralela ao eixo $\mathrm{Y}$ indica o limite superior para $\mathrm{k}=0$ (espécies ausentes). A curva X-X representa a porcentagem acumulada de individuos (escala superior).

muitos individuos entre as espécies sociais.

$\mathrm{Na}$ familia Apidae ocorreram dois picos de atividade, em janeiro e outubro, devido ao substancial aumento de S. flavisetis. Apidae esteve presente nas flores durante todo o ano, com declínio na abundância de indivíduos nos meses de março, junho e setembro, com principal atividade entre 08:00 e 13:00 hs, e pico das 11:00-12:00 (Fig. $3 \mathrm{~A}$ ). Se retirarmos $S$. flavisetis da análise, percebe-se que os Apidae estiveram mais ativos no início da manhã, principalmente entre 08:0009:00 hs, com atividade provavelmente influenciada pela temperatura. Algumas espécies foram representadas em quase todas as coletas: Trigona pallens (maiores picos em janeiro e fevereiro), Scaptotrigona flavisetis (picos em janeiro e outubro) e Apis mellifera (pico em abril) (Fig.3A).

Megachilidae esteve presente em quase todos os meses, não sendo registrado nenhum indivíduo nos meses de janeiro e novembro. A espécie mais abundante, Megachile 
(Sayapis) sp.2, foi coletada em quase todos os meses (pico em maio) e apresentou maior atividade entre $14: 00$ e $16: 00 \mathrm{hs}$ (Fig. 3C).

Os Halictidae, ausentes nos meses de fevereiro, julho e agosto, tiveram grande frequência em abril, devido a presença de Augochlora thalia, Augochloropsis sparsilis e A. crassigena. Apresentaram maior atividade en- tre 7:00 e 10:00 hs (Fig. 3B).

Os Andrenidae foram representados somente por Oxaea festiva nos meses de março, abril e junho (Fig.3D). No Maranhão, tem-se registro dessa espécie em São Luis, em vegetação de dunas (Albuquerque, 1998). Além de $O$. festiva também têm sido amostrados no Estado $O$. flavescens e Protomelliturga turnerae (Apocalypse, 1995;

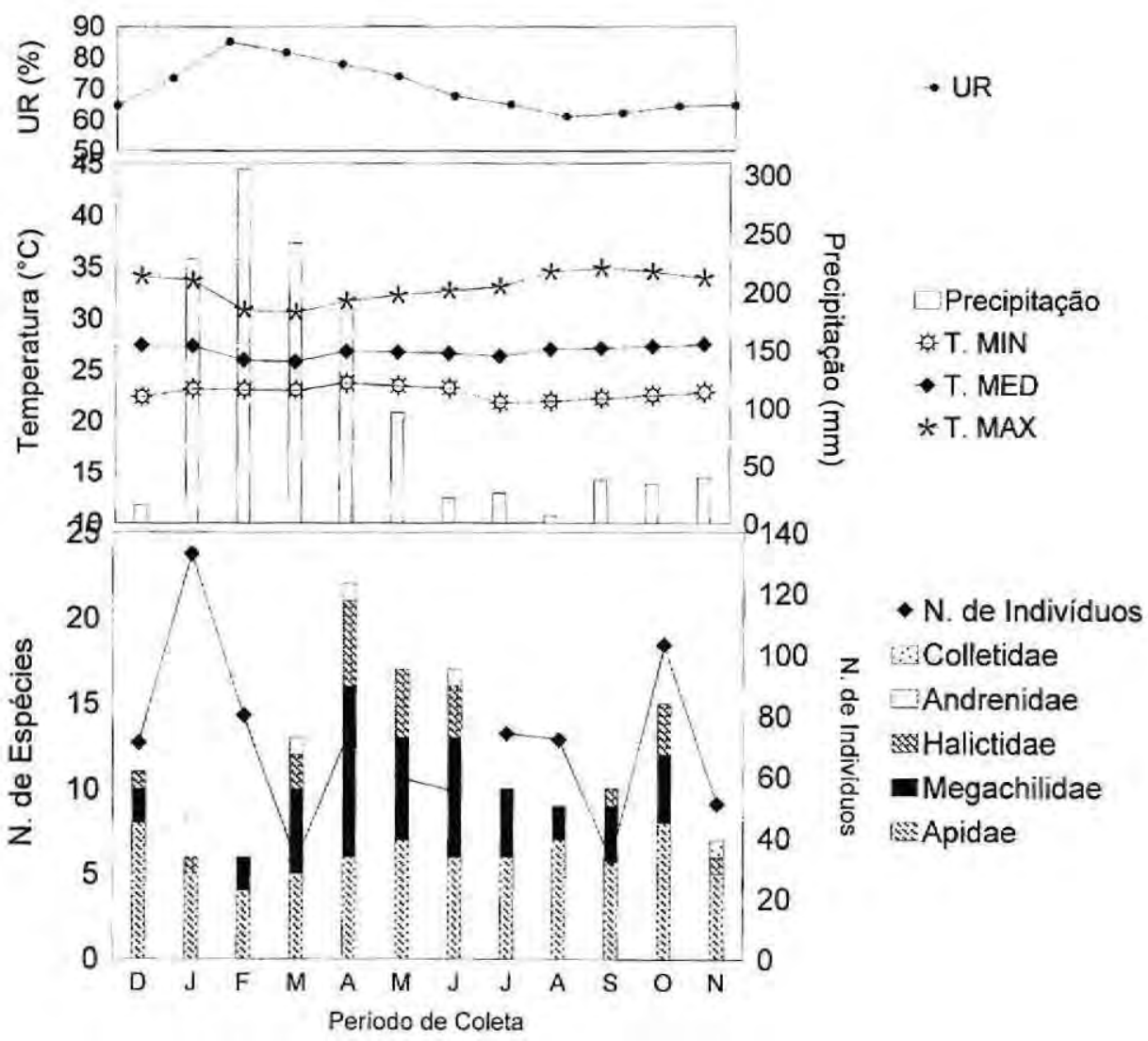

Figura 2. Dados climáticos - umidade relativa (UR) do ar, precipitação pluviométrica, temperaturas máxima (T.MAX), média (T.MED) e mínima do ar (T.MIN), abundância sazonal de espécies por familia de abelhas e número total de indivíduos coletados no município de Vitória do Mearim, MA, Brasil entre Dez/91 e Nov/92. 


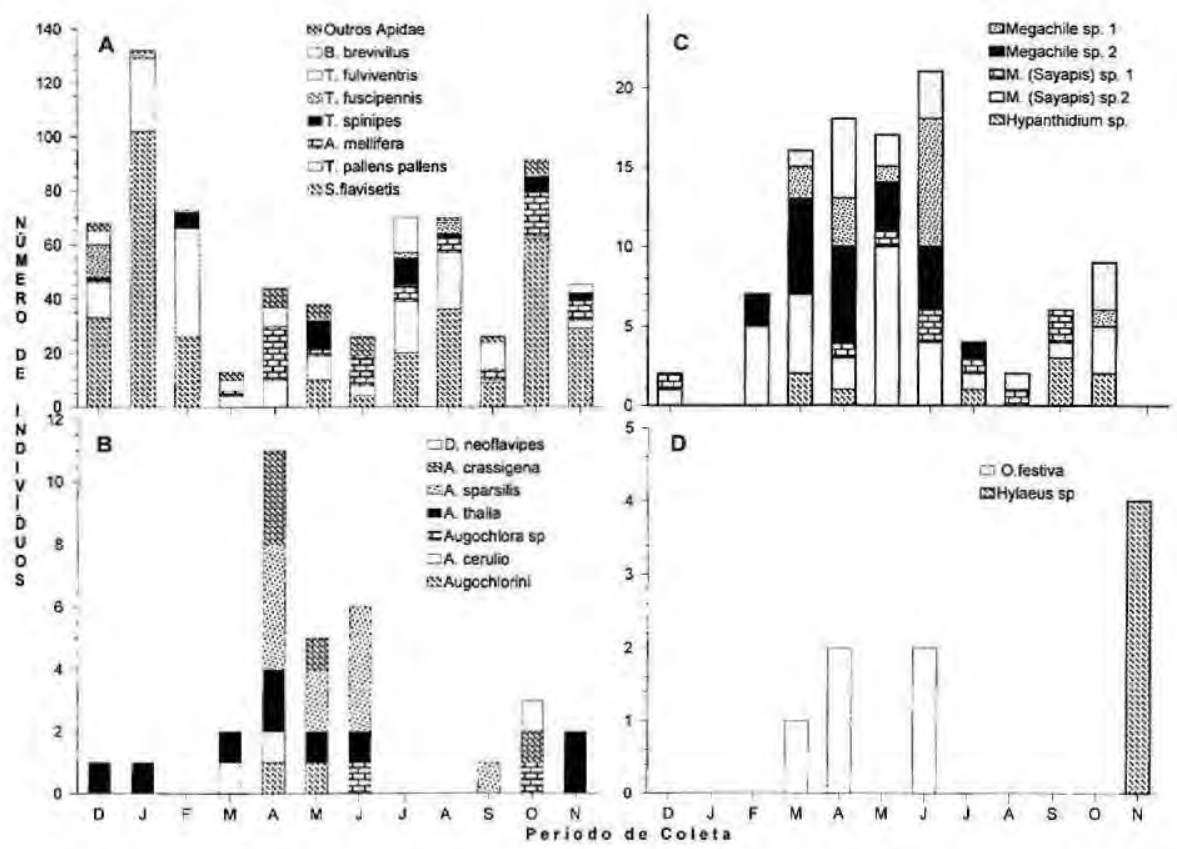

Figura 3. Número de individuos das espécies predominantes de Apidae (A), Halictidae (B), Megachilidae (C), Andrenidae (D) e Colletidae(D) coletados em Vitória do Mearim, MA, Brasil de Dez/91 a Nov/92.

Pereira, 1998).

Colletidae foi representado apenas por Hylaeus sp. coletado no mês de novembro (Fig. 3D). Este gênero parece ser o grupo que melhor representa a família no Maranhão (Brito, 1994; Araújo, 1994; Pereira, 1998).

As espécies mais abundantes de Apidae, coletadas em iscas odoriferas, foram frequentes em quase todos os meses: Eulaema (A.) nigrita (19 ind.; 26,4\%) atraida pela vanilina e eucaliptol, Euglossa (E.) cordata (18 ind.; $25 \%$ ) e Euglossa (E.) gr. modestior (14 ind.; $19,4 \%$ ), atraidas pelo eucaliptol. Eulaema nigrita possui dois picos de atividade, em abril e agosto; E. cordata apresentou pico de atividade também em agosto e em dezembro e E. modestior em julho. Já Eulaema meriana foi atraida pela vanilina e coletada somente no mês de julho. Euglossa (E.) mourei com registros apenas no Peru, Equador e Colômbia foi amostrada em Vitória do Mearim no mês de maio em eucaliptol. Eufriesea mussitans (salicilato de metila) é uma espécie sazonal, mostrando-se ativa somente no mês de fevereiro (Fig.4).

Das iscas odoriferas 
utilizadas, a que recebeu maior número de visitas foi eucaliptol, e isto se deu principalmente com as abelhas do gênero Euglossa (Fig. 4). Outros trabalhos realizados com Euglossinae em Manaus - AM (Braga, 1976; Morato et al., 1992), Salvador-BA (Raw, 1989), Cajuru - SP (Rebêlo \& Garófalo, 1991) e Barreirinhas - MA (Rebêlo \& Cabral, 1997) obtiveram resultados semelhantes, onde de todos os atrativos utilizados, o eucaliptol foi sempre o que capturou o maior número de indivíduos e espécies.

Apesar dos machos de Euglossinae visitarem as íscas durante todos os meses do ano (Fig. 5 ), as maiores frequências de visitas ocorreram no periodo seco (junho a dezembro; $58,3 \%$ ), o que contraria o que foi observado por Rebêlo \& Cabral (1997) em Barreirinhas (litoral ocidental MA), onde $71,6 \%$ dos individuos foi coletado na estação chuvosa.

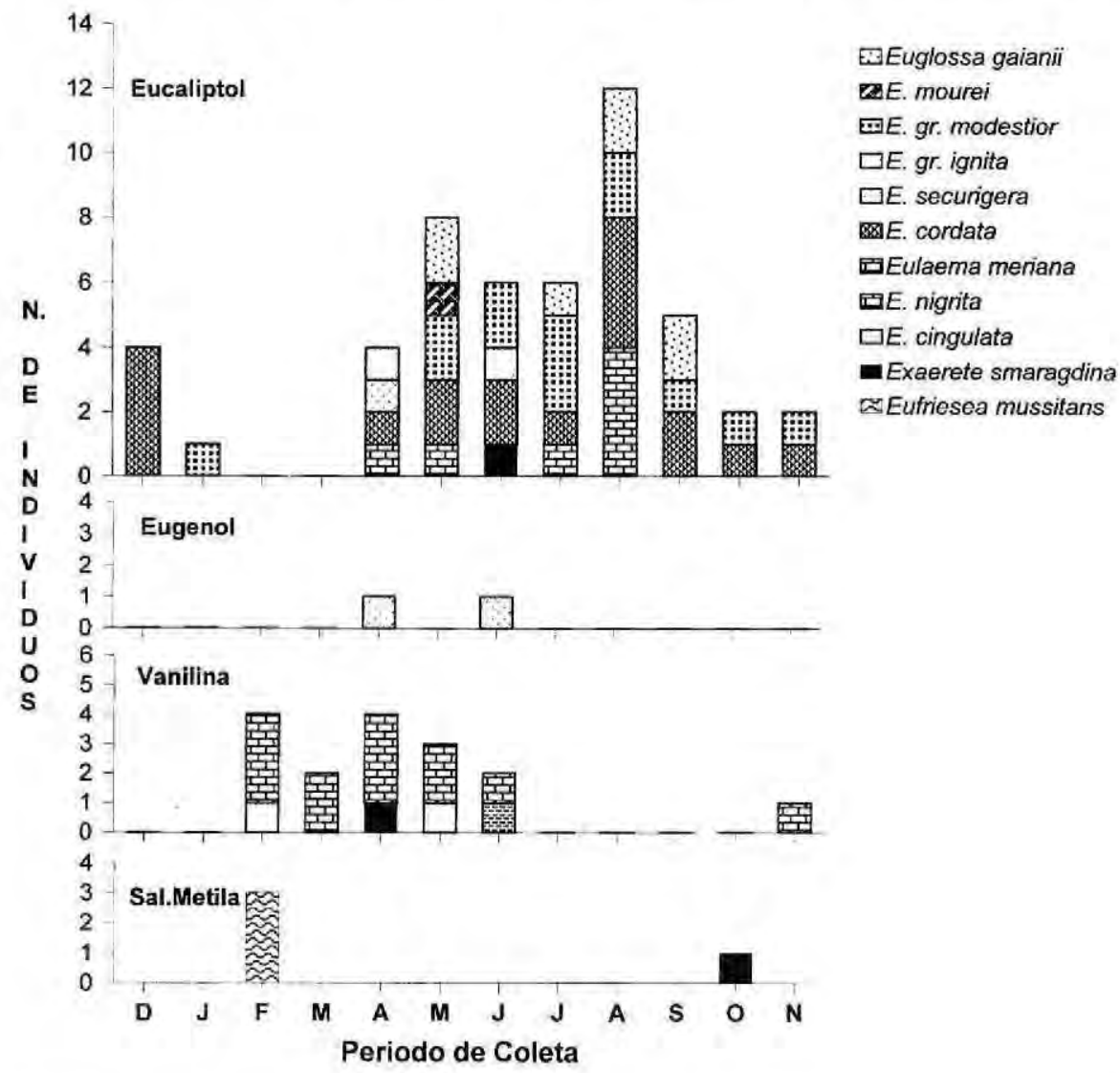

Figura 4. Número de individuos de Euglossinae coletados usando-se íscas-odoriferas em Vitória do Mearim, MA, Brasil de Dez/91 a Nov/92. 
Essa divergência pode ser devido ao fato de que, na Baixada Maranhense (Vitória do Mearim), a estação chuvosa tem a duração de apenas 5 meses enquanto no litoral o período chuvoso dura 8 meses.

Considerando-se o número de espécies como uma medida de diversidade, e fazendo-se uma comparação com outros levantamentos feitos no Maranhão e em outras localidades do Brasil, observamos que os inventários realizados no Maranhão apresentam uma baixa diversidade de espécies, assemelhando-se ao que foi encontrado em Casa Nova - BA (Fig. 6). Em relação a Apidae, porém, a diversidade é maior, semelhante a de Lençois BA e Ribeirão Preto - SP (Fig. 6). De acordo com CURE et al.
(1991), as espécies predominantes e sua abundância relativa variam entre áreas de uma mesma região biogeográfica; para termos uma idéia sobre a riqueza relativa em espécies, é necessário o levantamento de várias áreas dentro da mesma região. Isso nos leva a concluir que levantamentos padronizados de abelhas, constituem uma boa metodologia para estudos de relações entre flora e fauna, sobre as guildas estabelecidas, ou seja, como a comunidade está interagindo e porque variam numa mesma região, não são uma boa metodolgia para estimar riquezas entre regiões.

\section{AGRADECIMENTOS}

Os autores agradecem aos

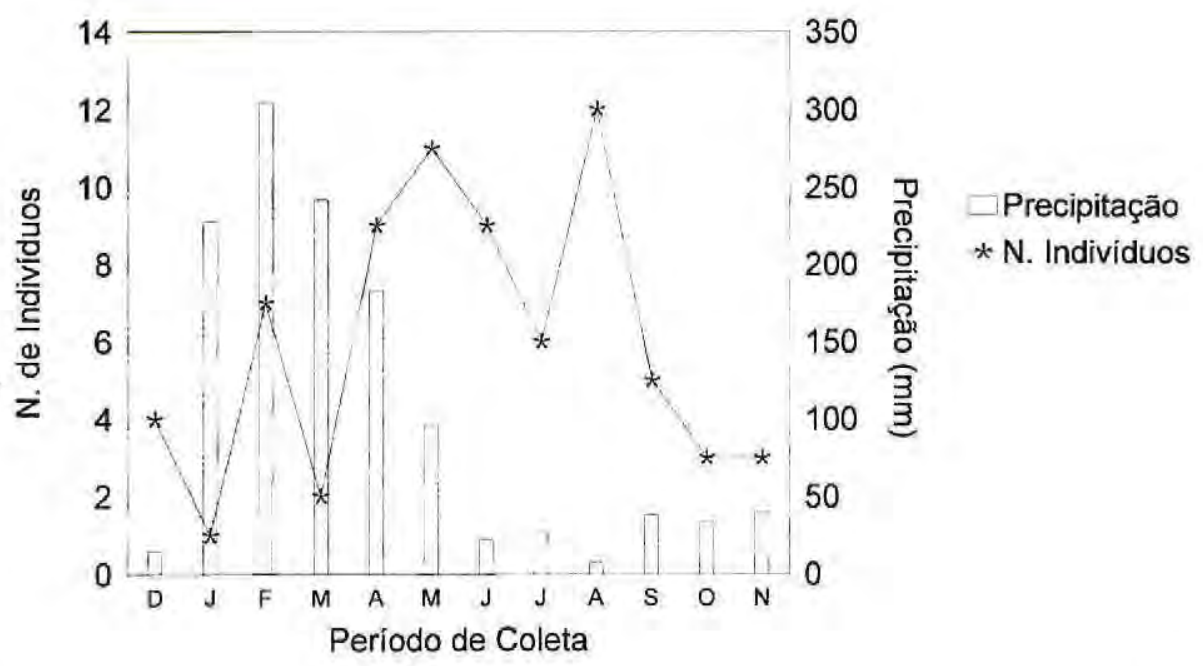

Figura 5. Fenologia dos machos de Euglossinae atraídos por íscas-odoriferas de Dez/91 à Nov/ 92, em Vitória do Mearim, MA, Brasil. 


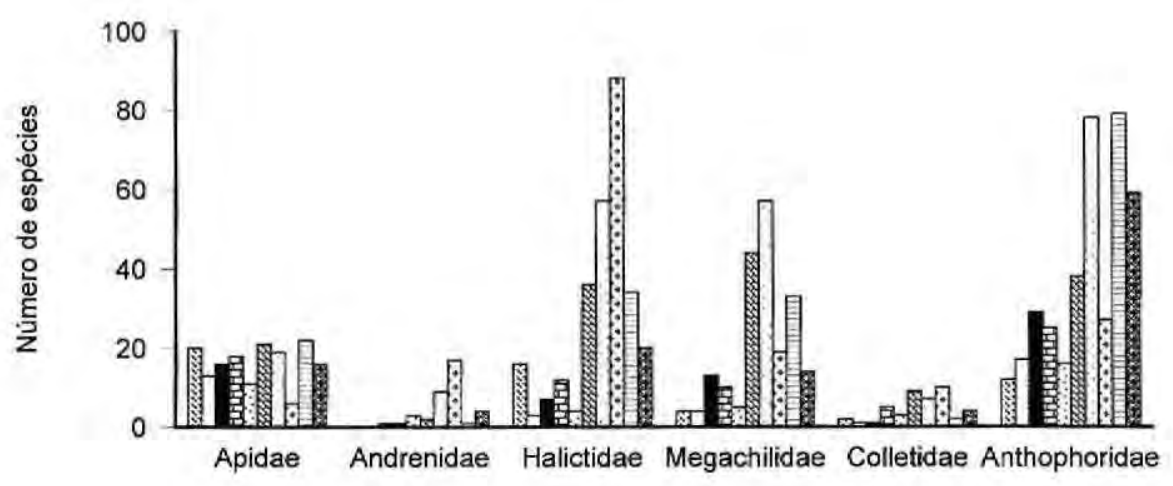

জ્alcântara (GONÇALVES et al., 1996)

$\square$ Barreirinhas (BRITO, 1994; ALBUQUERQUE \& MENDONÇA, 1996)

- Vitória do Mearim (Este trabalho)

퐁 Săo Luís (REBÊLO, 1996)

DCasa Nova (MARTINS, 1990; 1994)

mLençóis (MARTINS, 1990; 1994)

口Ribeirão Preto (CAMARGO \& MAZUCATO, 1984)

. José dos Pinhais (SAKAGAMI et al., 1967)

$\square$ Paraopeba (SILVEIRA \& CAMPOS, 1995)

\&Corumbatai (SILVEIRA \& CAMPOS, 1995)

Figura 6. Comparaçăo da diversidade das familias de abelhas coletadas em 10 localidades diferentes no Brasil.

Profs. João M.F. Camargo (FFCLRP-USP), Pe. Jesus Santiago Moure (UFPR), Danúncia Urban (UFPR) e José M.M. Rebêlo (UFMA) pela identificação das espécies; à Companhia Vale do Rio Doce (CVRD), Fundação de Amparo à Pesquisa do Maranhão (FAPEMA), projeto № 119/92 - DC e Conselho Nacional de Desenvolvimento Científico e Tecnológico (CNPq), proj. № 400675/91-9, pelo auxilio financeiro concedido.

\section{Bibliografia citada}

Ab'Saber, A.N. 1987. Aspectos geomorfológicos de Carajás. In:
Seminário sobre Desenvolvimento Econốmico e Impacto Ambiental em Áreas do Trópico Úmido Brasileiro. A experiência da CVRD, I., Belèm, Anais, CVRD, p. 201-232.

Albuquerque, P.M.C. ; Mendonça, J.A.C. 1996. Anthophoridae (Hymenoptera, Apoidea) e Flora associada em uma formação de cerrado no municipio de Barreirinhas, MA, Brasil. Acta Amazónica, 26(1/2):4554.

Albuquerque, P.M.C. 1998. Abelhas silvestres (Hymenoptera, Apoidea) e suas fontes de alimento em um ecossistema de dunas, na ilha do Maranhão, MA, Brasil: composição, fenologia e interações. Tese de Doutorado, Faculdade de Filosofia, Ciências e Letras de Ribeirão Preto, USP. Ribeirão Preto, São Paulo, 202 p.

Apocalypse, M. S. 1995. Inventario da Apifauna e flora associada (exceto Apidae) em região de capoeira, São Luis, 
MA, Brasil. Monografia de Graduação, Universidade Federal do Maranhão. São Luis, Maranhão. 50p.

Araujo, A. 1994. Halictidae, Anthophoridae, Megachilidae e Colletidae (Hymenoptera, Apoidea) em uma comunidade floristica, Alcântara, MA, Brasil. Monografia de Graduação, Universidade Federal do Maranhão. São Luís, Maranhão. 71p.

Bortoli, C. de; Laroca, S. 1990. Estudo biocenótico em Apoidea (Hymenoptera) de uma área restrita em São José dos Pinhais (PR, Sul do Brasil), com notas comparativas. Dusenia, 15:1-112.

Braga, P.I.S. 1976. Atração de abelhas polinizadoras de Orchidaceae com auxilio de íscas-odores na campina, campinarana e floresta tropical úmida da região de Manaus. Ciência e Cultura, 28:767-773.

Brasil. 1984. Instituto Brasileiro de Geografia e Estatística (IBGE). Atlas do Maranhão. Rio de Janeiro.

Brasil. 1991. Instituto Brasileiro de Meio Ambiente e dos Recursos Naturais Renováyeis (IBAMA). Secretaria de Estado do Meio Ambiente e Turismo do Maranhão (SEMATUR). Diagnóstico dos principais problemas ambientais do Estado do Maranhão.

Brito, C.M.S. de. 1994, Abelhas silvestres (Hymenoptera, Apoidea) e suas fontes de alimento no cerrado, Barreirinhas, MA, Brasil. Monografia de Graduação. Universidade Federal do Maranhão, São Luis, Maranhão. 69 p.

Camargo, J.M.F.; Mazucato, M. 1984. Inventário da apifauna e flora apicola de Ribeirão Preto, SP, Brasil, Dusenia, 14(2):55-87.

Campos,L.A.O.; Silveira, F.A. da; Oliveira, M.L.de; Abrantes, C.V.M.; Morato, E.F.; Melo, G.A.R. de. 1989. Utilizaçăo de armadilha para a captura de machos de Euglossini (Hymenoptera, Apoidea). Rev. brasil. Zool., 6(4):621-626.

Carvalho, A.M.C.; Bego, L.R. 1995. Seasonality of dominant species of bees in the Panga Ecological Reserve, cerrado, Uberlândia, MG. An.Soc.Entomol.Brasil.,
24(2):329-337.

Carvalho, A.M.C.; Bego, L.R. 1996. Studies on Apoidea fauna of cerrado vegetation at the Panga Ecological Reserve, Uberlândia, MG, Brazil. Rev. bras. Ent., 40(2):147156.

Cure, J.R. 1983. Estudo ecológico de comunidades de abelhas silvestres ( $\mathrm{Hy}$ menoptera, Apoidea) do Parque da Cidade, comparado ao de outras áreas de Curitiba, Paraná. Dissertação de Mestrado, Universidade Federal do Paraná. Curitiba, Paraná. 100p.

Cure, J.R.; Bastos Filho, G.S.; Oliveira, M.J.F. de; Souza, O.F. de. 1991. Influência do tamanho da amostra na estimativa da riqueza de espécies em levantamentos de abelhas silvestres (Hymenoptera, Apoidea). Revta.bras.Zool., 7(1-2):101110.

Gonçalves, S. de J.M.; Rego, M.M.C.; Araujo, A. de. 1996. Abelhas sociais (Hymenoptera: Apidae) e seus recursos florestais em uma região de mata secundária, Alcântara, MA, Brasil. Acta Amazônica. 26(1/2):55-68.

Kato, M.; Matsuda, T.; Yamasita, Z. 1952. Associative ecology of insects found in the paddy field cultivated by various planting forms. Sci. Rep. Tôhoku Univ., 4yh. Ser. (biology), 19(4):291-301.

Laroca, S. 1974. Estudo feno-ecológico em Apoidea do litoral e primeiro planalto paranaenses. Dissertação de Mestrado, Universidade Federal do Paraná. Curitiba, Paraná. 62p.

Laroca, S.; Cure, J.R.; Bortoli, C. 1982. A associação de abelhas silvestres ( $\mathrm{Hy}$ menoptera, Apoidea) de uma área restrita no interior da cidade de Curitiba (Brasil): uma abordagem biocenótica. Dusenia, 13(3):93-117.

Martins, C.F. 1994. Comunidade de abelhas (Hym., Apoidea) da caatinga e do cerrado com elementos de campo rupestre do Estado da Bahia, Brasil. Revta.Nord. Biol., 9(2):225-257.

Martins, C.F. 1995. Flora apícola e nichos tróficos de abelhas (Hym., Apoidea) na 
Chapada Diamantina (Lençóis-BA, Brasil). Revta.Nord.Biol., 10(2):119-140.

Morato, E.F.; Campos, L.A.O.; Moure, J.S. 1992. Abelhas Euglossini (Hymenoptera, Apidae) coletadas na Amazônia Central. Revta.bras.Ent., 36(4):767-771.

Pereira, C.Q.B. 1998. Inventário da Apifauna em uma área de reserva florestal préamazônica, Buriticupu-Maranhão-Brasil. Monografia de Graduação, Universidade Federal do Maranhão. Sao Luis, Maranhão. 31 p.

Raw, A. 1989. The dispersal of Euglossini bees between isolated patches of eastern brazilian wet forest (Hymenoptera, Apidae). Revta.bras.Ent., 33(1):103-107.

Rebêlo, J.M.M. 1995. Espécies de Anthophoridae (Hymenoptera, Apoidea) e sua associação com flores, numa área restrita da ilha de São Luís-MA, Brasil. Bol.Mus.Para.Emilio Goeldi, sér.Zool., 11(2): 105-124.

Rebêlo, J.M.M; Garofalo, C.A. 1991. Diversidade e sazonalidade de machos de Euglossini (Hymenoptera, Apidae) e preferências por iscas-odores em um fragmento de floresta no sudeste do Brasil. Rev. bras. Biol., 51(4):787-799.

Rebêlo, J.M.M.; Cabral, A. de J.M. 1997. Abelhas Euglossinae de Barreirinhas, zona do litoral da baixada oriental maranhense. Acta Amazónica, 27(2):145-152.

Règo, M.M.C. 1998. Abelhas silvestres (Hym., Apoidea) em um ecossistema de cerrado s.l. (Chapadinha - MA, Brasil): uma abordagem biocenótica. Tese de Doutorado, Faculdade de Filosofia, Ciências e Letras de Ribeirão Preto, USP. Ribeirão Preto, São Paulo. 181 p.

Sakagami, S.F,; Laroca, S.F. 1971. Relative abundance, phenology and flower visits of apid bees in Eastern Paraná, southern Brazil (Hymenoptera, Apidae). Kontyü, 39(3):217-230.
Sakagami, S.F.; Laroca, S.; Moure, J.S. 1967. Wild bee biocenotics in São José dos Pinhais (PR), South Brazil Preliminary report. J.Fac.Sci.Hokkaido Univ., sér. 6, Zoology, 18:57-127.

Schlindwein, C. 1998. Frequent oligolecty characterizing a diverse bee-plant community in a xerophytic bushland of subtropical Brazil. Stud.Neotrop.Fauna \& Environm., 33:46-59.

Silveira, F.A.; Campos, M.J.O. 1995. A melissofauna de Corumbatai (SP) e Paraopeba (MG) e uma análise da biogeografia das abelhas do cerrado brasileiro (Hymenoptera, Apoidea). Revta. bras. Ent., 39(2):371-401.

Taura, H.M.; Laroca, S. 1991. Abelhas altamente sociais (Apidae) de uma área restrita em Curitiba (Brasil); distribuição dos ninhos e abundância relativa. Acta Biol. Par, 20(1/2/3/4):85-101.

Wilms, W.; Imperatirz-Fonseca, V.L.; Engels, W. 1996. Resource partitioning between highly eusocial bees and possible impact of the introduced africanized honey bee on native stingless bees in the Brazilian Atlantic Rainforest. Stud. Neotrop. Fauna \& Environm., 31:137-151.

Wittmann, D.; Hoffman, M. 1990. Bees of Rio Grande do Sul, southern Brazil (Insecta, Hymenoptera, Apoidea). Theringia, sér. Zool., 70:17-43,

Zanela, F.C.V.; Schwartz Filho, D.; Laroca, S. 1998. Tropical bee island biogeography: diversity and abundance patterns. Biogeographica, 74(3):103-115. 